\title{
Application of Silicon Photomultipliers to Calorimetry and Positron Emission Tomography
}

\author{
Martin Göttlich * \\ DESY, Notkestr. 85, D-22607 Hamburg, Germany \\ E-mail: martin.goettlich@desy.de

\section{Erika Garutti} \\ DESY, Notkestr. 85, D-22607 Hamburg, Germany \\ E-mail: erika.garutti@desy.de
}

\author{
Alexander Tadday ${ }^{\dagger}$ \\ University of Heidelberg, Im Neuenheimer Feld 227, D-69120 Heidelberg, Germany \\ E-mail: alexander.tadday@kip.uni-heidelberg.de \\ Hans-Christian Schultz-Coulon \\ University of Heidelberg, Im Neuenheimer Feld 227, D-69120 Heidelberg, Germany \\ E-mail: coulon@kip.uni-heidelberg.de
}

\begin{abstract}
Silicon Photomultipliers (SiPMs) are novel photon counting devices consisting of multiple Avalanche Photodiodes operating in Geiger mode. Their excellent photon counting capability and their compact design make them very promising candidates for the direct read-out of organic and inorganic scintillators in order to construct detectors with unprecedented granularity. We discuss both their application to calorimetry and to Positron Emission Tomography.
\end{abstract}

International Workshop on New Photon Detectors PD09

June 24-26 2009

Shinshu University Matsumoto Japan

*Corresponding author

† Speaker 


\begin{tabular}{|l|l|l|l|l|l|}
\hline $\begin{array}{l}\text { \# Pixels } \\
\left(\text { size }\left[\mathrm{mm}^{2}\right]\right)\end{array}$ & $\begin{array}{l}\text { Bias } \\
{[\mathrm{V}]}\end{array}$ & $\begin{array}{l}\text { Dark Rate } \\
(0.5 \text { p.e. threshold })\end{array}$ & $\begin{array}{l}\text { Pixel Size } \\
{\left[\mu \mathrm{m}^{2}\right]}\end{array}$ & $\begin{array}{l}\text { Gain } \\
\left(10^{5}\right)\end{array}$ & $\begin{array}{l}\text { PDE } \\
{[\%]}\end{array}$ \\
\hline $1600(1 \times 1)$ & 70.4 & 200 & $25 \times 25$ & 2.5 & 25 \\
\hline $3136(1.4 \times 1.4)$ & 71.8 & $550\left(200 \mathrm{kHz} / \mathrm{mm}^{2}\right)$ & $25 \times 25$ & 2.5 & 25 \\
\hline $400(1 \times 1)$ & 69.4 & 600 & $50 \times 50$ & 7.5 & 50 \\
\hline $676(1.3 \times 1.3)$ & 70.5 & $900\left(530 \mathrm{kHz} / \mathrm{mm}^{2}\right)$ & $50 \times 50$ & 7.5 & 50 \\
\hline $3600(3 \times 3)$ & 68.5 & $4600\left(511 \mathrm{kHz} / \mathrm{mm}^{2}\right)$ & $50 \times 50$ & 7.5 & 50 \\
\hline \hline $\begin{array}{l}2 \times 2 \text { matrix } \\
4 \times 3600(3 \times 3)\end{array}$ & 68.5 & $4500\left(500 \mathrm{kHz} / \mathrm{mm}^{2}\right)$ & $50 \times 50$ & 7.5 & 50 \\
\hline
\end{tabular}

Table 1: Properties of the MPPC devices used in the present study. Dark rate, gain and photon detection efficiency (PDE) are given at nominal voltage. The PDE quoted here is the peak value (in the blue spectral region) and includes effects of crosstalk and afterpulses.

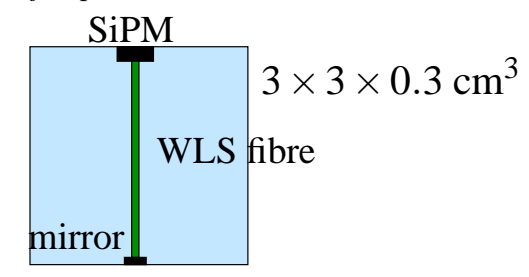

Figure 1: Tile with SiPM, WLS fibre and mirror.

\section{Introduction}

The Silicon Photomultiplier (SiPM) is a novel type of photon-counting device made of multiple avalanche photodiode pixels operated in Geiger mode ([1],[2]). SiPM devices offer an excellent photon-counting capability and are easy to operate. They reach a gain up to $10^{6}$ with a relatively low bias voltage supply ranging typically from $30 \mathrm{~V}$ to $80 \mathrm{~V}$. The resulting current signal is sizable even for a single detected photon and has a rise time of less than 100ps leading to an excellent time resolution. Furthermore they are insensitive to magnetic fields, small sized and can be read out with simple electronics. These properties make them very promising candidates for highly granular detectors. The Multi-Pixel Photon Counter (MPPC) tested in this work has been developed by HAMAMATSU [3]. It is a kind of SiPM, but unlike the first devices of this family, they provide very good sensitivity in the blue spectral region (cf. [4]). This matches the peak emission wavelength of common organic and inorganic scintillators. This improves both the energy and the time resolution and allows the direct read-out of scintillators with high efficiency. In the present article we discuss the application of MPPC devices to calorimetry and to Positron Emission Tomography. Table 1 summarises the most important properties of the MPPC devices used in the present study.

\section{Calorimetry Application}

With the goal of developing a highly granular hadronic calorimeter we use SiPM devices to read out $3 \times 3 \mathrm{~cm}^{2}$ plastic scintillator tiles. Tiles with and without WLS fibre are investigated. The figures of merit are the number of photons which are detected when a minimal ionising particle is 
a)

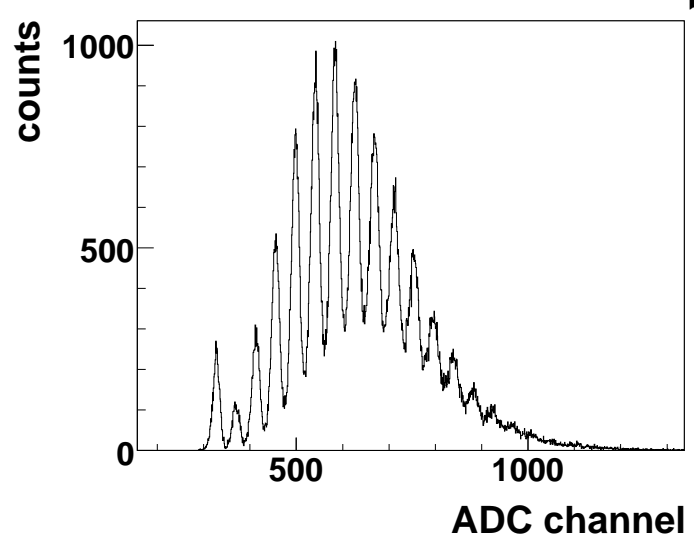

b)

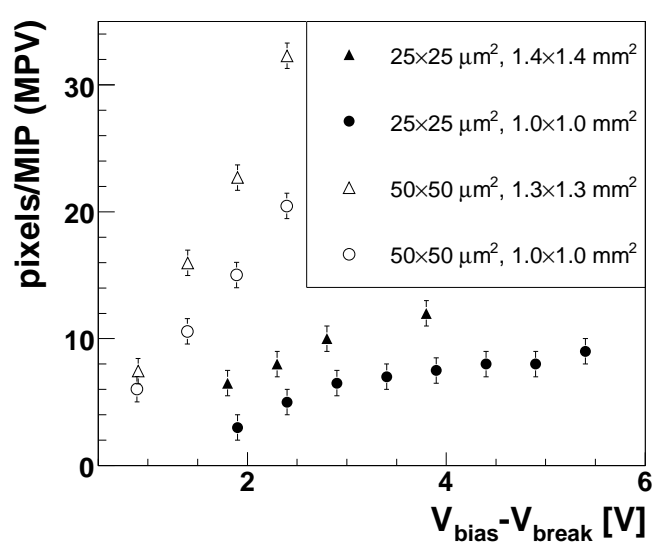

Figure 2: a) Typical MIP spectrum for a MPPC device with a pixel size of $50 \times 50 \mu m^{2}$. b) Pixels/MIP $(M P V)$ as a function of the overvoltage for different MPPC devices (direct read-out).

going through the tile and the detection efficiency. We discuss and compare the results we get for MPPC devices of different types and sizes (see table 1). As a source for minimal ionising electrons ${ }^{90} \mathrm{Sr}$ is used. Two different $3 \times 3 \times 0.3 \mathrm{~cm}^{3}$ plastic scintillator tiles (manufactured by Uniplast enterprise) are investigated. One of the tiles contains a green wavelength shifting fibre (Kuraray multicladding WLS fibre Y11(200)) of a $0.5 \mathrm{~mm}$ radius (see figure 1). The second tile has no WLS fibre installed and is used to test the direct read-out of the blue scintillation light. Both tiles are wrapped in a Super-radiant VN2000 foil $(3 M)$. The signal integration is done by a QDC (Lecroy 1182 ), using a gate of $80 \mathrm{~ns}$. The QDC is triggered by an additional scintillator with photomultiplier tube read-out which is installed behind the tested tile/MPPC system w.r.t. the electron flux.

Figure 2a) shows a typical MIP spectrum for a MPPC device with a pixel size of $50 \times 50 \mu \mathrm{m}^{2}$. Each peak corresponds to a certain number of pixels firing in the MPPC. The first peak is the pedestal. The MIP spectra are used to derive the most probable value (MPV) for the number of pixels fired per MIP. We test different MPPC devices for the direct read-out of scintillator tiles. The results in terms of pixels/MIP (most probable value, MPV) are depicted in figure $2 b$ ). Due to the larger photon detection efficiency (PDE) we observe a higher light yield for devices with a pixel size of $50 \times 50 \mu \mathrm{m}^{2}$. Furthermore we see an increase of the light for devices with a larger area. The increase scales with the area. In figure 3 we compare the light yield per MIP for direct and WLS fibre read-out, respectively. Only the devices with the larger area are considered here. The light yield is significantly larger for WLS fibre read-out since the light is collected very efficiently by the fibre and directed to the sensor. Furthermore, for WLS fibre read-out the light yield is expected to depend less on the actual position where the particle hits the tile. On the other hand, tiles with WLS fibres installed are more complex to build and thus more expensive which is an important factor for a calorimeter consisting of a few million channels (about 10000 channels $/ \mathrm{m}^{3}$ ). The direct read-out is an important step towards simplifying the system and reducing the overall costs. In order to judge if direct read-out is feasible in spite of the smaller light yield per MIP we determined 
a)

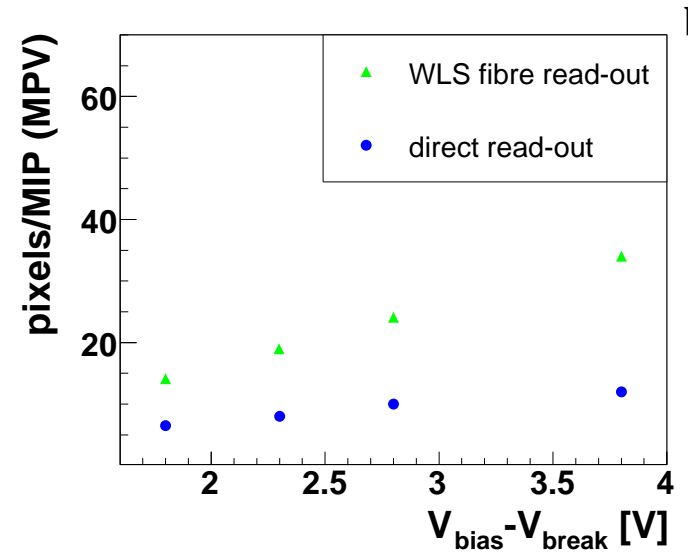

b)

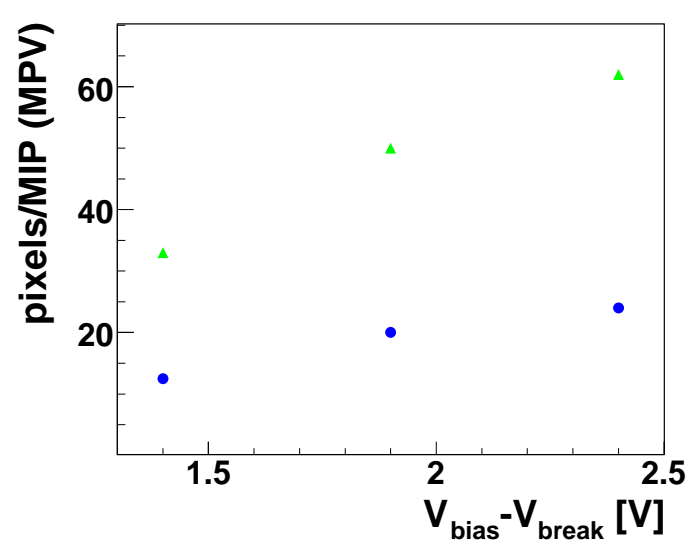

Figure 3: Pixels/MIP as a function of the overvoltage for WLS and direct read-out. a) MPPC device with a pixel size of $25 \times 25 \mu \mathrm{m}^{2}$ and an active area of $1.3 \times 1.3 \mathrm{~mm}^{2}$. b) MPPC device with a pixel size of $50 \times 50 \mu \mathrm{m}^{2}$ and an active area of $1.4 \times 1.4 \mathrm{~mm}^{2}$.

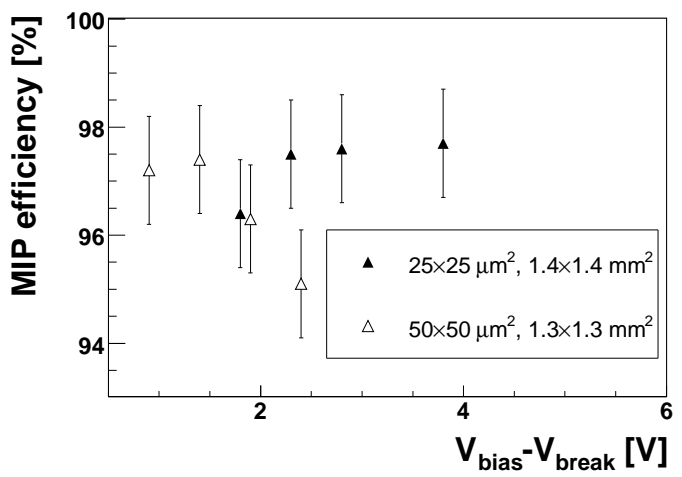

Figure 4: MIP efficiency as a function of the overvoltage.

the efficiency to detect a MIP. In practice the noise threshold has to be chosen such that per event only one of the 10000 channels $/ \mathrm{m}^{3}$ is triggered by SiPM noise. The efficiency is defined as number of events above the noise threshold devided by the total number of events. Figure 4 shows the efficiencies as a function of the overvoltage. For the voltage ranges considered here the efficiencies are large enough for both types of MPPC devices. To avoid large saturation corrections the device with the smaller pixel size and hence the larger number of pixels is the best option.

\section{Application to Positron Emission Tomography}

Apart from the calorimeter application we study the prospects of using Silicon Photomultipliers for medical imaging modalities such as Positron Emission Tomography scanners. Single crystal $\left(3 \times 3 \times 15 \mathrm{~mm}^{3}\right.$ LSO [5] and LFS [6] crystals read out by $3 \times 3 \mathrm{~mm}^{2}$ MPPC) studies with SiPM read-out show very promising results in terms of energy (11-12\% FWHM for $511 \mathrm{keV}$ photons) and coincidence time resolution (470 ps FWHM) as we discussed in [7]. Therefore, we constructed 
a)

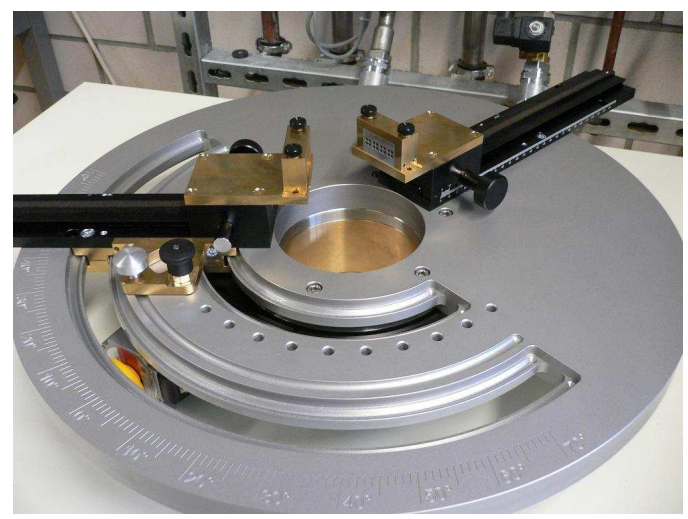

b)

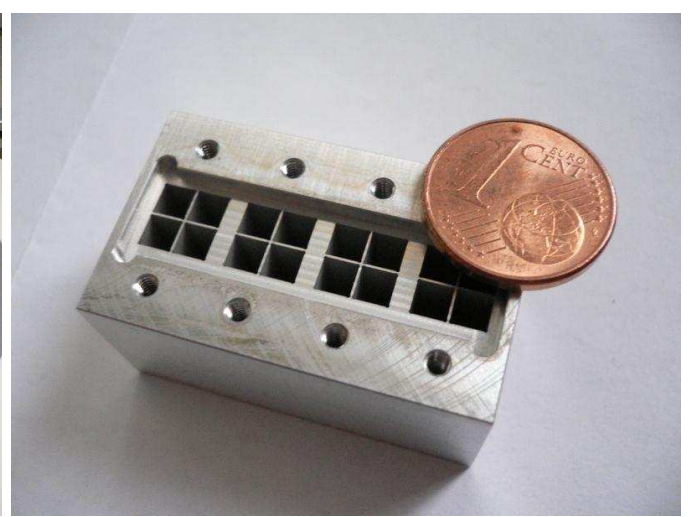

Figure 5: a) PET test-device. b) Module consisting of four submodules with four crystals read out individually by a $2 \times 2$ MPPC array.

a 32-channel prototype scanner with a pixel-size of $3 \times 3 \mathrm{~mm}^{2}$ which will be tested at DESY. The purposes of this prototype are manifold:

- The prototype will serve as a proof of concept.

- It will enable us to test different options for the multi-channel read-out which are scalable to a larger scanner for commercial use.

- The calibration and monitoring requirements of a multi-channel detector need to be addressed as well as the stability of operation.

- We will have a test-device for other camera configurations, e.g. multi-layer configurations.

- We will be able to perform studies towards a TOF-PET scanner.

The prototype is shown in figure 5a). The scanner consists of two modules. In order to detect all possible lines of response the modules are rotated around the centre of the field of view by means of a high precision rotation stage. In addition each module can be individually moved in radial direction and the angle between the modules can be changed. Each module consists of four submodules. A submodule is a $2 \times 2$ matrix of $3 \times 3 \times 15 \mathrm{~mm}^{3}$ LFS crystals which are read out by a $2 \times 2$ MPPC array from Hamamatsu. Each MPPC is read out individually, for a total number of 16 channels per module. It is forseen to build two additional modules to reduce the scan time which will give 64 read-out channels in total. The modules (see figure $5 \mathrm{~b}$ ) are made of aluminium. The cavities for the crystals are eroded by electrical discharge machining. The individual crystals are separated by a $100 \mu \mathrm{m}$ thick wall. The aluminium provides a very good reflectivity, hence, a good energy resolution is achieved as it is depicted in figure 6. For the individual channels we get an energy resolution of $11 \%$ to $12 \%$ FWHM for $511 \mathrm{keV}$ photons from a ${ }^{22} \mathrm{Na}$ source. The photoelectric peak is clearly separated from the Compton-continuum. The energy resolution is extracted by a Gaussian fit to the peak region. The ratio of the FWHM and the mean of the fit (subtracting the pedestal) is quoted as an estimate for the energy resolution. 


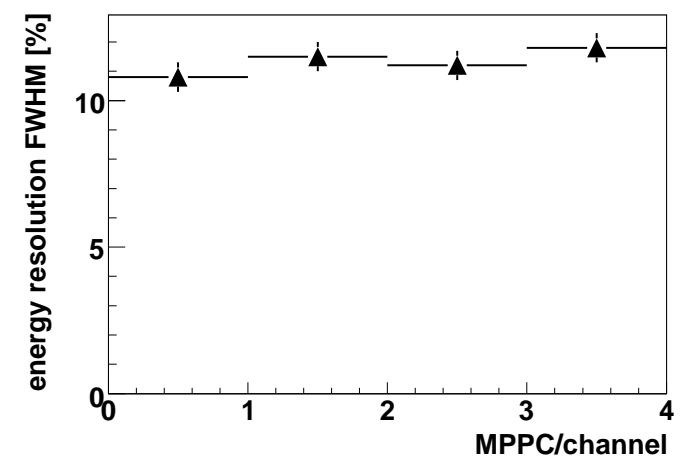

Figure 6: Energy resolution for each channel of a submodule.

In order to predict the performance of the PET test-device in terms of spatial resolution, Monte Carlo simulation studies are performed using the GATE toolkit [8] which is based on GEANT4 [9]. Applying the Filtered Backprojection algorithm for image reconstruction we expect a spatial resolution of $2.6 \mathrm{~mm}$ FWHM (see [7]). Simulation studies show that with a smaller pixel size, e.g. $1 \times 1 \mathrm{~mm}^{2}$, a resolution below one millimetre can be achieved.

For the multi-channel read-out we consider two options. In the first approach we consider to use the NINO ASIC, an ultra fast low power front end amplifier discriminator chip [10] which is a development of the CERN electronics department. The NINO ASIC delivers time and energy information via the time-over-threshold method. The NINO output is processed by a multi-hit TDC module (CAEN V1290A).

The University of Heidelberg developed a current mode constant fraction discriminator ASIC. A high-pass-filter scheme is implemented with $0.35 \mu \mathrm{m}$ CMOS technology to produce the bipolar signal shape for the time pick-off which has been proven to be as good as the conventional delayline scheme. Thanks to the fast signal processing capability of current-mode circuits, a small time walk of only $100 \mathrm{ps}$ for an input Range of 5:1 and a time jitter less than $20 \mathrm{ps}$ has been guaranteed by the simulation [11].

\section{Conclusions}

The Multi-Pixel Photon Counter is well suited for the direct read-out of organic and inorganic scintillators due to its peak sensitivity in the blue spectral region. The high efficiency in the spectral region where scintillators have their peak emission leads to a high light yield. Together with the relatively low noise of the MPPC devices this leads to an advantageous signal-to-noise ratio in the case of direct read-out of scintillator tiles. The conclusions of our studies are that among the tested devices the MPPCs with a pixel size of $25 \times 25 \mu \mathrm{m}^{2}$ are the best option for the direct read-out of plastic scintillator tiles. A signal-to-noise ratio larger than seven can be obtained with proper voltage adjustment, which is sufficient for an accurate MIP detection with an efficiency around $97 \%$. They provide a significantly larger dynamic range than the devices with a pixel size of $50 \times 50 \mu \mathrm{m}^{2}$ which is very important for the calorimetry application. However, the issue of non-uniformity of the response has to be investigated. 
For the PET application a large light yield due to the blue sensitivity is beneficial for both the energy and time resolution. In an earlier study we obtained very promising results for the energy (11-12\% FWHM) and coincidence time resolution (470 ps FWHM) for the crystal/MPPC system; the first result was reobtained for the individual channels of a $2 \times 2$ crystal/MPPC matrix. This motivates the next step towards a PET test-device. We are now on the verge of commissioning the prototype. This will enable us to address questions on the stability of operation, calibration issues and multi-channel read-out solutions. Furthermore we will be able to test different camera designs and to perform studies towards a TOF-PET system. This could in principle include tests on new scintillating crystals, photodetectors and electronics.

\section{Acknowledgements}

This work is supported by the Helmholtz-Nachwuchsgruppen grant VH-NG-206 and the BMBF, grant no. 05HS6VH1.

\section{References}

[1] P. Buzhan et al., "Silicon photomultiplier and its possible applications", Nucl. Instrum. Meth. A $\mathbf{5 0 4}$ (2003) 48.

[2] A. N. Otte et al., "A test of silicon photomultipliers as readout for PET", Nucl. Instrum. Meth. A 545 (2005) 705 .

[3] Hamamatsu, "Multi-Pixel Photon Counter", Product information, www.hamamatsu.com

[4] Alexander Tadday et al., "Characterisation Studies of Silicon Photomultiplier for a Calorimeter for the ILC", International Workshop on New Photon Detectors PD09, Proceedings of Science

[5] Hilger Crystals, "LSO Scintillation Material", Product Information, www.hilger-crystals.co.uk

[6] Zecotek, "LFS Scintillation Material", Product Information, www.zecotek.com

[7] M. Göttlich, E. Garutti, H.C. Schultz-Coulon, A. Tadday, "Application of Multi-Pixel Photon Counter to Positron Emission Tomography”, Nuclear Science Symposium Conference Report, 2008. NSS '08 IEEE, 19-25 Oct. 2008, Pages: 3119-3122

[8] S. Jan et al., "GATE : a simulation toolkit for PET and SPECT", Phys. Med. Biol. 49, 4543 (2004) [arXiv:physics/0408109]

[9] S. Agostinelli et al. [GEANT4 Collaboration], "GEANT4: A simulation toolkit", Nucl. Instrum. Meth. A $506(2003) 250$.

[10] F. Anghinolfi, P. Jarron, A. N. Martemyanov, E. Usenko, H. Wenninger, M. C. S. Williams and A. Zichichi, "Nino: An Ultra-Fast And Low-Power Front-End Amplifier/Discriminator Asic Designed For The Multigap Resistive Plate Chamber”, Nucl. Instrum. Meth. A 533 (2004) 183.

[11] Wei Shen et al., "Current mode Constant Fraction Discriminator for PET using SiPM (MPPC)", To be presented at the NSS '09 conference, Poster Number N13-36 